Nervenarzt 2008 [Suppl 3] · 79:113

DOI 10.1007/s00115-008-2568-1

(c) Springer Medizin Verlag 2008

\author{
W. Maier ${ }^{1} \cdot \mathrm{H} . \mathrm{Sa}^{2}$ \\ ${ }^{1}$ Klinik und Poliklinik für Psychiatrie und Psychotherapie, Universitätsklinikum, Bonn \\ 2 Universitätsklinikum, Aachen
}

\title{
Zertifizierte Fortbildung zum DGPPN-Kongress 2008
}

\author{
Demenz - von der Grundlagenforschung bis zu \\ Therapieansätzen in fortgeschrittenen Stadien
}

Das diesjährige CME-Supplement zur psychiatrischen-psychotherapeutischen Fort- und Weiterbildung anlässlich des jährlichen DGPPN-Kongresses ist 2008 der Diagnosegruppe der „Demenzen“ gewidmet.

Demenzen stellten früher ein vernachlässigtes Gebiet der medizinischen Versorgung und der klinischen Forschung dar. Die aktuelle und vor allem zukünftige Relevanz dieses medizinischen Arbeitsfeldes ist seit wenigen Jahren enorm gestiegen. $\mathrm{Zu}$ verdanken ist diese neue Akzentsetzung einerseits der Wahrnehmung der drohenden Gesundheitsgefahren aufgrund der demographischen Entwicklung und andererseits den aus einer blühenden Grundlagenforschung zur Neurodegeneration abgeleiteten neuen Perspektiven für Diagnostik und Therapie. Dieses CMEHeft versucht entsprechend einen breiten Bogen zu spannen zwischen einer Summe aus wichtigen Ergebnissen aus der Grundlagenforschung vor allem zur AlzheimerErkrankung und praxisnah entwickelten, nichtpharmakologischen Therapieansätzen in fortgeschrittenen Demenzstadien.

Von der Grundlagenforschung (Beitrag Prof. Bayer, Göttingen) angestoßen, hat in den vergangenen Jahren die Entwicklung von Biomarkern für eine frühzeitigere und sichere Diagnose bei Demenzen eine stürmische Entwicklung genommen. Heute kann man unterscheiden zwischen klinischen demenziellen Syndromen, die durch Gedächtnisbeeinträchtigungen und andere kognitive Störungen bestimmt werden (in ICD-10 oder DSM-IV definiert) und den zugrunde liegenden, meist viele Jahrzehnte vorher be- ginnenden neurodegenerativen Veränderungen des Gehirns (v. a. bei AlzheimerKrankheit). Die Spuren dieses vorauseilenden biologischen Krankheitsprozesses sind mit heute verfügbaren technischen Mitteln schon sehr frühzeitig, teilweise sogar schon vor den ersten Gedächtnisproblemen darstellbar: durch die Analyse des Liquors und durch verschiedene Methoden der Bildgebung. Der neueste Stand dieser Frühdiagnostik wird in den Beiträgen von Dr. Jessen, Bonn, und Prof. Wiltfang, Essen dargelegt.

Die in zwei Jahrzehnten gewonnenen Erkenntnisse aus der Grundlagenforschung haben ebenso zu einer stürmischen Entwicklung von neuen potenziellen Wirkmechanismen gegen die Alzheimer-Erkrankung geführt. Das Ziel ist dabei nicht nur eine vorübergehende symptomatische Verbesserung (wie diese $z$. B. bei den gegenwärtig verfügbaren Acetylcholinesterasehemmern der Fall ist). Vielmehr wird das direkte Eingreifen in den der Demenzentwicklung zugrunde liegenden neurodegenerativen Krankheitsprozess angestrebt. Mehrere solcher innovativen Ansatzpunkte für Therapieentwicklungen sind in den vergangenen Jahren entwickelt und validiert worden. Besonderes Interesse und besondere Hoffnung liegen vor allem auf verschiedenen Strategien der Impfung, die in diesem Heft stellvertretend für andere, neue potenzielle Mechanismen stehen (Beitrag Prof. Dodel, Marburg).

Auf absehbarer Zeit ist nicht zu erwarten, dass alle Formen der Demenzen vor Ausbruch der Krankheitssymptomatik gestoppt oder in einem frühen Stadium aufgehalten werden können. Es wird also auch weiterhin einen erheblichen Bedarf an Therapiemethoden geben, die nicht nur die kognitive Symptomatik betreffen, sondern auch die im Laufe der Demenzerkrankung zunehmend auftretenden psychologischen und Verhaltensprobleme, die für Patienten, Pflegende und Behandler eine besonders starke Belastung darstellen. Hier sind die verfügbaren medikamentösen Möglichkeiten begrenzt. Erfreulicherweise wurden in den vergangenen Jahren aber wirksame nichtmedikamentöse Therapieverfahren entwickelt, die auch in der häuslichen und institutionellen Pflege eingesetzt werden können (Beitrag Prof. Hüll, Freiburg). Die nichtmedikamentösen Therapieverfahren können die Belastung für Pflegende und ggf. auch den Pflegebedarf merklich reduzieren und die Lebensqualität steigern.

Wir wünschen dieser CME-Ausgabe eine ähnlich hohe Akzeptanz wie den beiden Vorgängern zur „Depression“ und „Schizophrenie“. Den Lesern wünschen wir eine interessante, gewinnbringende Lektüre und Erfolg bei der Beantwortung der CME-Fragen.

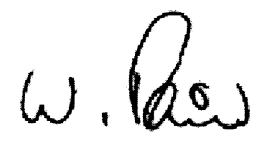

Prof. Dr. W. Maier, Bonn

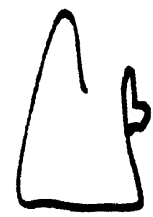

Prof. Dr. H. Saß, Aachen 